Mechanical and Surface Chemical Analysis of Retrieved Breast Implants from a

\title{
Single Centre
}

Louise J Magill ${ }^{1}$, Aleksandra Tanska ${ }^{1}$ Mohammed Keshtgar ${ }^{1}$, Ash Mosahebi ${ }^{1,2}$,

Gavin Jell ${ }^{1}$

1: Division of Surgery and Interventional Science, Royal Free Campus, University

College London, Pond Street, London NW3 2QG.

2: Department of Plastic and Reconstructive Surgery, Royal Free London NHS

Foundation Trust, Pond Street, London, NW3 2QG

Key words: Breast Implant, Silicone Implant, Breast Reconstruction, Failure

Financial Support: nil

\section{Corresponding author:}

Louise J Magill,

Division of Surgery and Interventional Science, Royal Free Campus, University

College London,

$9^{\text {th }}$ Floor Royal Free Hospital,

Pond Street,

London,

NW3 2QG.

Email: louise.magill.14@ucl.ac.uk 


\begin{abstract}
Introduction: Breast implants are associated with complications such as capsular contracture, implant rupture and leakage often necessitating further corrective surgery. Re-operation rates have been reported to occur in up to $15.4 \%$ of primary augmentation patients and up to $27 \%$ in primary reconstructions patients within the first three years [1]. The aim of this study was to examine the mechanical and surface chemical properties as well as the fibroblast response of retrieved breast implants in our unit to determine the in vivo changes which occur over time.
\end{abstract}

Methods: Ethical approval was obtained. 21 implants were retrieved. Implantation time ranged from 5 months -300 months (Mean 133.4 months). Tensile strength, elongation, Young's modulus and tear strength properties were measured using Instron 5565 tensiometer on anterior and posterior aspects of the implant. Attenuated total reflectance-fourier transform infra-red spectroscopy (ATR-FTIR), wettability and scanning electron microscopy (SEM) analysis was performed on the shell surfaces. Bicinchoninic acid assay was performed to determine shell protein content. The fibroblast response was determined by seeding HDFa cells on the retrieved implants and cell metabolism measured using Alamar Blue ${ }^{\mathrm{TM}}$ assay.

Results: Mechanical properties fall with increasing duration of implantation. There were no significant changes in ATR-FTIR spectra between ruptured and intact implants nor significant changes in wettability in implants grouped into 5 year categories. SEM imaging reveals surface degradation changes with increasing duration of implantation.

Conclusions: With increasing duration of implantation, mechanical properties of the breast implants fall. However this was not associated with surface chemical changes as determined by ATR-FTIR and wettability nor protein content of the shells. Thus the reduction in mechanical properties is associated with breast implant failure but further research is required to elucidate the mechanisms. 


\section{Introduction}

Breast implants have a role in aesthetic and reconstructive breast surgery however little is known of the mechanism of aging and in vivo response to breast implants. Complication rates or need for re-operation within the first three years following initial implant surgery have been reported up to $15.4 \%$ in primary augmentation patients and up to $27 \%$ in primary reconstruction patients [1]

Silicone gel breast implants are composed of an elastomer shell envelope containing a gel made from the polymer polydimethylsiloxane ( $\left.\mathrm{PDMS}\left(\mathrm{CH}_{3}\right)_{2} \mathrm{SiO}\right)$. The difference between the shell and gel composition is the degree of cross-linking between the polymer chains [2]. The mechanism of breast implants failure during implantation resulting in capsular contracture (a pathological fibrous encapsulation of the implant), gel bleed or leaking of silicone into the surrounding tissues is not known. In addition, the rupture mechansim of implants is not fully elucidated.

Several studies have sought to establish the cause of breast implant failure. Research has shown that increasing implantation times negatively effect the mechanical strength properties in explanted breast implants [3-5]. Furthermore, mechanical weakening of the shells have been also postulated to be attributed to swelling of the breast implant shells with low molecular weight silicones diffusing from the gel into the shells during implantation [2,6]. Attenuated total reflectance-Fourier transform infra-red spectroscopy (ATR-FTIR) evaluates the chemical composition and quantity of molecules in any given substance using infrared radiation to identify chemicals and chemical bonds. ATR-FTIR has been studied in breast implants highlighting potential functional groups with may interact with the surrounding host cellular environment [7] and surface chemical changes of the implants following radiation therapy [8] and implantation [9]. Prasad et al [10] demonstrated that increasing the surface roughness of silicone elastomers samples produced a decrease in fibroblast growth which may account for reduced incidence of capsular contracture using textured rather than smooth breast implants [11]. Valencia-Lazcano et al [12] showed that increased surface roughness of uninplanted breast implants resulted in greater fibroblast adhesion in an in-vitro model. However, no studies to our knowledge have investigated fibroblast behavior on retrieved breast implants. 
Thus, the aim of this study was to characterize the effects of implantation and aging on the mechanical and surface chemical properties of the implants retrieved from patients attending for elective removal or exchange of implants and their in vitro fibroblast response. 


\section{Materials and Methods}

\section{Consent and Patient recruitment}

All patients attending for exchange or removal of breast implants were included in the study. Two patients attending for unilateral exchange of breast implant had undergone breast radiation therapy prior to their implant reconstruction which may have increased their reconstruction failure risk. Patients were consented by the author prior to surgery. Breast implants were collected from theatre, labeled anonymously, visually inspected and cleaned with iso-propanolol. The implants were cut using a scalpel into anterior shell and posterior shells and the inner gel carefully inspected and removed. The shells were then cleaned with iso-propanolol and left to air dry at room temperature prior to analysis.

\section{Tensile Testing and Tear Testing}

From the implant shells, six dumbbell shaped (20mm x 4mm) specimens were cut using a Wallace cutting press and three crescent shaped specimens for tear testing from both the anterior and posterior parts of the implant shell in accordance with the ISO 37:2005 standards. The location of where the specimens where taken from each shell is shown in Figure 1. Specimens were placed in the pneumatic grips of the tensiometer (Instron 5565 tensiometer equipped with a $500 \mathrm{~N}$ load, Instron, UK) and pulled apart at a rate of $100 \mathrm{~mm} / \mathrm{min}$ and $500 \mathrm{~mm} / \mathrm{min}$ for tensile and tear testing respectively. The data was captured using Bluehill software. Ultimate tensile strength, strain at break, Young's modulus and tear strength values were recorded.

\section{ATR-FTIR of Breast Implant Shells}

The midpoint of the anterior shell of each implant was tested $(n=5)$. The breast implant shells were quantified using ATR-FTIR recordings using Jasco FT/IR 4200 Spectrometer with a diamond attenuated total reflectance accessory (Diamond Miracle ATR, Pike Technologies, US). A spectrum was produced ( $n=5)$ from an average of 30 scans a spectrum was produced over a range of $600 \mathrm{~cm}^{-1}$ to $4000 \mathrm{~cm}^{-1}$ with a resolution of $4 \mathrm{~cm}^{-1}$. A background scan was performed prior to every measurement. 


\section{Surface wettability contact angle measurements $(\theta)$}

Using a DSA 100 Krüss Goniometer, wettability analysis was performed on the breast implants outer shell surface. Using the sessile drop technique, $5 \mu l$ of deionized water was dropped onto the implants using an automated syringe with 10 seconds of dispensing and analysis was performed using the Drop Analysis software $(n=12)$ (EasyDrop DSA200, KrüSS) at room temperature.

\section{Scanning Electron Microscopy of Breast Implant Shells}

Breast implant shell specimens were immersed in 1\% Triton X100 and 1\% sodium dodecyl sulfate for 16 hours, washed $\mathrm{x} 2$ with deionized water then washed with absolute ethanol followed by a further $\mathrm{x} 2$ washes with deionized water to remove any biological proteins. The samples were then dried in a 40 degrees oven for 1 hour. The samples were then mounted on aluminum stubs using carbon adhesive tabs and sputter coated with gold/palladium using a High Resolution Ion Beam Coater (Gatan Model 681). Images were taken using a scanning electron microscopy at magnifications ranging from x50 to x 1000 using a Field Emission Scanning Microscope (JEOL- JSM 7401F).

\section{Protein quantification assay (Bicinchoninic Acid (BCA) assay) of breast implant specimens.}

The protein content of the breast implant shells specimens were analysed using the Bicinchoninic (BCA) assay. 0.25gram of implant shell and 0.25grams of gel were placed in $0.2 \%$ trifluoroacetate and acetonitrile 50v/50v for 24 hours in a dark cupboard at room temperature. The solution was evaporated to dryness using a speedivac, the implant shell removed and the extracted proteins were re-suspended in $250 \mu \mathrm{l}$ of water. Protein content of the resuspension was determined using the Pierce $^{\text {TM }}$ BCA Protein assay kit, (BCA assay) ThermoFisher Scientific, UK. The absorbance was measured at $592 \mathrm{~mm}$ excitation using a fluorescent plate reader (Fluoroskan Ascent FL ${ }^{\mathrm{TM}}$, ThermoScientific ${ }^{\mathrm{TM}}$, USA)

\section{Cell culture and seeding}

Human dermal fibroblasts (HDFa) were cultured in Dulbecco's Modified Eagle's Medium, low glucose (Gibco, ThermoScientific ${ }^{\mathrm{TM}}$, UK) supplemented with 10\% FBS (foetal bovine serum) and 1\% PenStrep (penicillin-streptomycin, Gibco). HDFa's used in the experiment were between passage 7 and 11. 
For seeding experiments, $6 \mathrm{~mm}$ discs of breast implants shells were decellularised by placing in $1 \%$ Triton $\mathrm{X}$ for $1 \mathrm{hr}$, washing twice in PBS followed washing in $70 \%$ ethanol followed by washing twice in PBS. Discs were then placed in a 96 well plate and covered with $100 \mu$ l of warmed DMEM for approximately 2 hours prior to cell seeding. Each disc was seeded with HDFa cells at density of $5 \times 10^{4}$ cells $/ \mathrm{cm}^{2}$.

\section{Cell metabolism}

In order to assess cell metabolic activity Alamar Blue ${ }^{\mathrm{TM}}$ assay (Invitrogen, Paisley, UK) was used. Cells seeded onto tissue culture plastic served as a positive control and media only wells provided a negative control. Cells were incubated at $37^{\circ} \mathrm{C}$ at $5 \%$ $\mathrm{CO} 2$ in air and cell culture media was replenished on days 0, 1, 3 and 6. Cell metabolism was assessed using Alamar Blue ${ }^{\mathrm{TM}}$ assay according to manufacturer's guidelines on days 1, 3 and 7. Media from the wells was removed and fresh media containing $10 \%$ Alamar Blue ${ }^{\mathrm{TM}}$ solution was added to each well. Following a $4 \mathrm{hr}$ incubation wrapped in aluminium foil, $100 \mu \mathrm{l}$ of the media from each well was placed into a 96-well plate and analysed using a fluorescent plate reader (Fluoroskan Ascent FL ${ }^{\text {TM }}$ Fluorescence Plate Reader, ThermoScientific, USA) at an excitation and emission wavelengths of $530 \mathrm{~nm}$ and $620 \mathrm{~nm}(\mathrm{n}=6)$.

\section{Statistics}

All statistics were performed using either linear correlation, non-parametric Spearman correlation, one-way ANOVA and 2 way ANOVA where significance was $\mathrm{p}<0.05$. All graphs were performed using GraphPad Prism software Version 6 apart from ATR-FTIR were presented using Microsoft Excel. 


\section{Results}

A total of 15 patients were recruited to the study. All patients were female and mean age at time of implant removal or exchange was 42.3 years (SD 8.36 years). Eleven patients had undergone breast implant surgery for reconstructive purposes ( 9 unilateral procedures, 2 bilateral procedures) and four patients had undergone breast implant surgery for cosmetic augmentation (4 bilateral procedures). Three of fifteen patients were smokers. Two patients had undergone breast radiation therapy prior to their implant reconstruction ( 2 unilateral procedures, 2 implants). Twenty-one breast implants and 8 corresponding samples of surrounding capsular issue were retrieved. All implants retrieved featured a textured surface. The mean time from initial operation to removal or exchange of implant was 133.3 months (SD 90.1 months). Five implants (24\%) were ruptured at time of retrieval. The reasons for removal were capsular contracture (suffering either Baker III or IV level of capsular contracture) (5 implants), exchange for permanent implant (5), suspected or confirmed rupture (4), pain/discomfort (3), complication with other breast (2), symmetrisation (1), presence of axillary silicone granuloma (1). A summary of patient and breast implant characteristics is provided in Table 1.

Retrieved gels varied in colour from clear, colourless to strong yellow. Ruptured implants contained strong yellow discoloured gels in comparison to intact implants.

\section{Implant Type and Manufacturer}

Implants collected were found to be from a range of manufacturers and included Allergan (5), McGhan (3), Mentor (2), Poly Implant Prothèsis (3) and Labaratoire Sebbin (2). Five of the 21 retrieved implants did not exhibit manufacturers details and were therefore labeled as 'unknown'. Furthermore, implants were determined as tissue expanders or permanent implants as shown in Table 2. 


\section{Tensile Mechanical Properties of Retrieved Implants}

A significant fall in ultimate tensile strength (UTS) is observed with increasing implantation times both for anterior shell $(\mathrm{p}=0.0003, \mathrm{r}-0.708)$ and posterior shells specimens from the explanted implants $(\mathrm{p}=0.0312, \mathrm{r}=-0.5085)$ as shown in Fig. $2 \mathrm{~A}$ and B. In addition, Young's modulus for anterior shell specimens $(\mathrm{p}=0.0037$, $\mathrm{r}=0.6049$ ) was reduced however, posterior shell specimens showed no significant difference $(p=0.2032, r=-0.3149)$ as shown in Fig. 3. Strain at break demonstrated a significant reduction in anterior shell specimens $(\mathrm{p}=0.0003, \mathrm{r}=-0.7158)$ but no significant difference was observed in the posterior shell specimens $(\mathrm{p}=0.0654, \mathrm{r}=-$ 0.4433) as shown in Fig. 4.

\section{Tear Strength of Retrieved Implants}

Ultimate tear strength demonstrated a significant reduction with increasing implantation times as shown in Fig. 5A and B for anterior $(p=0.0006, r=-0.6714)$ and posterior shell specimens $(\mathrm{p}=0.0254, \mathrm{r}=-0.5108)$

\section{ATR-FTIR of Retrieved Implant Shells}

Chemical analysis of the retrieved breast implant shells grouped into 5 year categories was determined by ATR-FTIR, outlined in Fig. 6A. The average spectrum of implant shells $(n=5)$ grouped into 5-year categories is shown in the overlaid spectra in Fig. 6B and Fig. 6C.

On review, there was statistically significant observed changes at the peak spectral height $784 \mathrm{~cm}^{-1}$ corresponding to $-\mathrm{CH}_{3}$ rocking and $-\mathrm{Si}-\mathrm{C}$-stretching in $-\mathrm{Si}-\mathrm{CH}_{3}$ as shown in Fig. 7A ( $\mathrm{p}=0.0224$, one-way ANOVA, parametric data). This may be however simply an effect of changes in the implant's thickness and elasticity rather than true degradation changes. However, there were no significant differences between the peak spectral heights at $1004 \mathrm{~cm}^{-1}$ corresponding to the asymmetric stretching of -Si-O-Si- (Fig. 8B p=0.2152, one-way ANOVA, parametric data) and at $1257 \mathrm{~cm}^{-1}$ corresponding to symmetric bending of $-\mathrm{CH}_{3}$ in $-\mathrm{Si}-\mathrm{CH}_{3}$ (Fig. $7 \mathrm{C}$ $\mathrm{p}=0.1698$, one-way ANOVA, parametric data). Furthermore, on statistical analysis of the peak spectral height between the intact group $(n=16)$ versus ruptured shells $(n=5)$ found no significant differences as shown in Fig. 8. 


\section{Surface wettability/Contact Angle measurements ( $\boldsymbol{\theta}$ )}

There was no significant difference in contact angle/surface wettability in the implants grouped into 5-year duration of implantation categories as shown in Fig. 9 $(\mathrm{p}=0.7583$. one way ANOVA).

\section{Scanning Electron Microscopy of Breast Implant Shells}

The SEM images of retrieved implants from the same manufacturer of differing implantation times demonstrated within increasing implantation times the breast implant shell surface was roughened with pits, grooves and 'ragged' edges.

All retrieved implants irrespective of duration of implantation displayed evidence of surface wearing. The McGhan ${ }^{\mathrm{TM}}$ implants retrieved after 85 months (Fig. 10A) and 110 months (Fig. 10B) showed increasing evidence of ragged edges of the manufactured pits with surface dents and grooves. In the Allergan ${ }^{\mathrm{TM}}$ implants retrieved after 7 months (Fig. 10C) and 204 months (Fig. 10D) surface degradation was evident with ragged edges, increasing irregularity of the pits outline more pronounced in the 204 months implant suggesting increasing surface degradation with increasing duration of implantation.

\section{Protein quantification of Implant Shells - Bicinchoninic Acid (BCA) Assay}

Volume of extracted protein from retrieved breast implant shells varied significantly as determined by Bicinchoninic Acid (BCA) assay. There was no statistically significant difference determined between protein content of the shells and duration of implantation ( $\mathrm{p}=0.91$, two tailed correlation) as shown in Fig. 11.

\section{Alamar Blue ${ }^{\text {TMAssay }}$ (Cytotoxicity and Cell Metabolism)}

HDFa cells were cultured for 7 days and Alamar Blue ${ }^{\mathrm{TM}}$ assays were performed at 24 hours, 72 hours and 7 days as shown in Figure 12.. There were significant differences at Day 1 between TCP and Allergan ${ }^{\mathrm{TM}} 7$ months and McGhan ${ }^{\mathrm{TM}} 85$ months only. At Day 3 there were significant reduced cell metabolism on all implants apart from McGhan 85 months. At Day 7 there was significantly increased metabolism in all implants apart from McGhan ${ }^{\mathrm{TM}} 110$ months. Analysis of the time points revealed significant differences between the timepoints $(\mathrm{p}<0.0001)$ and between the groups $(\mathrm{p}$ $=0.003,2$ way ANOVA, multiple comparisons) 


\section{Discussion}

At the time of inception in 1962, the breast implant manufactured by Dow Corning Corporation featured a thick silicon envelope and viscous inner gel that ultimately led to high rupture and silicone gel bleed rates. After five generations of manufacturing adjustments the current fifth generation breast implants are comprised of a textured surfaced silicone elastomer envelope containing a cohesive silicone gel to minimize capsular contracture, gel leakage and implant rupture rates. All patients who were approached participated in the study agreed to take part. Increasing implantation times demonstrated a significant reduction in the shells ability to withstand stretch both for the anterior and posterior components of the implant. In addition, tear strength was significantly reduced with increasing implantation times. This is in-keeping with previous work by Greenwald et al. [3] of 25 retrieved implants who showed that increasing implantation times resulted in reduced shell strength and elasticity $[4,13,14]$. In addition, Brandon et al. [15] showed, in un-implanted control breast implants, evidence of significant lot to lot variability as well as between different models of implant by same manufacturer suggesting variability in the manufacturing process of implants.

Swelling of the breast implant shells caused by diffusion of low molecular weight silicones from the gel have been implicated in weakening the mechanical properties $[2,6]$. Lipid infiltration to the shells has also been implicated in causing degradation of breast implant shells in vivo [16]. In addition, our study showed that contact angle measurements/wettability was not statistically changed with increasing duration of implantation. Previous work by Wei et al. [17] demonstrated that increasing hydrophilic surfaces promote cell adhesion in a mouse fibroblast model and hydrophobic surfaces promote cell spreading. Valencia-Lazcano et al. [12] examined new un-implanted implants from a range of manufacturers and reported contact angles for textured implant surfaces between 130 and 142 degrees in-keeping with our work. Furthermore, protein deposition levels in this study showed no significant changes in relation to the implantation times. 
ATR-FTIR analysis showed significant changes only at the peak spectral height 784 $\mathrm{cm}^{-1}$ corresponding to $\mathrm{CH}_{3}$ rocking and $-\mathrm{Si}-\mathrm{C}$-stretching in $-\mathrm{Si}-\mathrm{CH}_{3}$. This may represent degradation of the surface of the implant however, there was no significant differences seen in the other peak spectral heights nor was there any statistically significant differences demonstrated in all peak spectral heights comparing the ruptured and intact shells. The changes witnessed may also reflect changes in the elasticity and thickness of the breast implant specimens tested. The spectra and peak intensities produced are in keeping with previous work by Yildrimer et al [9]. However, they found there was a statistically significant difference in peak intensity at $1007.6 \mathrm{~cm}^{-1}$ corresponding to stretching of the Si-O-Si polymer when comparing explanted PIP implants containing industrial grade silicone and explanted implants produced by Allergan and Mentor manufacturers which may have influenced their results.

Gel colour changes identified on naked eye inspection from clear, colourless gels in intact, short duration of implantation implants to deeply yellow gels and in one case cloudy gels in older and ruptured implants is in-keeping with previous work [14]. The cause of this is yet unknown but has been postulated to be an unknown biological component which diffuses from the host through the silicone elastomer to interact with the gel. However, this does not uniformly occur in all explanted implants [14]. Yildirimer et al. [9] demonstrated protein-like peaks intensities on FTIR analysis of gels from ruptured implants suggesting a bacterial contaminent. Further work analyzing the gels of explanted breast implants revealed evidence of lipid infiltration [16] but no evidence of protein or peptides in the gels from intact implants sampled by proteonomic analysis [18]. A further area of study would be to analyse the colour intensity of the retrieved gels and correlate this with the implant shell mechanical properties.

Scanning electron microscopy imaging revealed visual changes in the surface of the implants with roughening of the pits and ragged edges. Comparing retrieved breast implant shells from same manufacturer, through scanning electron microscopy there was evidence of increasing surface degradation with increasing implantation times. However, further research is required to quantify this further using retrieved implants from a single manufacturer and type and a range of implantations times to further detect the true effect of in vivo aging. In addition, a possible explanation may be trauma to the implant at time of retrieval surgery and separation from the surrounding 
encapsulated implant could account for the surface changes encountered as shown in cadaver studies [19].

The cellular response results show no significant differences in cell metabolism between the implants tested but increased with each timepoint. For the purpose of this study we used human dermal fibroblasts to establish the cellular response, however, future research is required examining the behavior of other cell types as well as measurement of inflammatory cytokines would be of value.

The limitations of our study included retrieval of breast implants from a range of different manufacturers and small sample size. This small sample size may be reflected in this was performed as an exploratory study of all patients presenting to a single unit and potential reluctance of patients to present to medical services with breast implant-related morbidity' In addition, the implants were retrieved from patients who had originally undergone insertion of implants for both received breast implants both for augmentation and for reconstructive purposes which may have also influenced the results. Two of sixteen patients had undergone pre-operative radiation therapy prior to implant placement and this may have contributed to increased risk of capsular contracture and implant failure as previously been reported [20].

\section{Conclusion}

This study reported a significant fall in mechanical properties of the breast implants shells with increasing duration of implantation. Surface chemical properties were largely unchanged. Further research is required to elucidate the mechanisms of breast implant failure. 
